\title{
EGFR tyrosine kinase inhibitor therapy for lung cancer treatments and their clinical outcomes: A cohort study in Taiwan
}

\author{
CHING-HU CHUNG
}

\begin{abstract}
Department of Medicine and Institute of Long-Term Care, Mackay Medical College, New Taipei 252, Taiwan, R.O.C.
\end{abstract}
Received November 5, 2018; Accepted August 29, 2019

DOI: 10.3892/ol.2019.10942

\begin{abstract}
Malignant cancer is the top cause of mortality in Taiwan. In particular, the mortality rate of with lung cancer reached 39.2/100,000 in 2017. Epidermal growth factor receptor tyrosine kinase inhibitors (EGFR-TKIs) are being increasingly used to treat lung cancer.; however, due to small sample sizes and a limited number of adequately controlled studies, it is difficult to compare survival rates of traditional chemotherapy with EGFR-TKI therapy when used as a first- or second-line treatment for patients with lung cancer, and therefore data on its efficacy are inconclusive. Therefore, Taiwan's entire 2010-2015 National Health Insurance Database (NHID) was used to perform a retrospective study. The top two anti-neoplastic first-line therapies used for lung cancer were traditional platinum-based doublet chemotherapy and EGFR-TKI therapy. Patients with stage III and IV lung cancer undergoing first-line EGFR-TKI therapy exhibited improved overall survival rates. However, patients with stage I and II lung cancer demonstrated limited benefits. Patients with stage IIIB and IV EGFR mutation (-) patients did not benefit from treatment with EGFR-TKI therapy. The EGFR-TKI gefitinib may be more effective in patients with lung cancer than erlotinib, irrespective of whether patients had been previously treated or not. Patients treated with Gefitinib also exhibited improved survival rates compared with other frequently used chemotherapeutic drugs.
\end{abstract}

\section{Introduction}

Lung cancer is one of the most common types of malignant cancer and the leading cause of cancer-associated death in Taiwan, United States and the European Union $(1,2)$. Wang et al (3) have shown that the 5-year overall survival (OS) rate for all patients with lung cancer in Taiwan is $15.9 \%$,

Correspondence to: Professor Ching-Hu Chung, Department of Medicine and Institute of Long-Term Care, Mackay Medical College, Building 46, Section 3, Zhongzheng Road, Sanzhi, New Taipei 252, Taiwan, R.O.C.

E-mail: chchung@mmc.edu.tw

Key words: lung cancer, prevalence, tyrosine kinase inhibitors, gefitinib, Taiwan being relatively lower in patients with late-stage lung cancer (4.9\% in stage IV). For a large proportion of patients, the disease is initially diagnosed at a later stage of cancer, and drug treatment is required for long-term survival improvement. Typically, systemic therapy is used in patients with advanced or recurring disease following initial definitive treatment. The therapeutic regimen used depends on the stage of the cancer, the molecular characteristics of the tumour and the patient's overall medical condition. Advances in drug treatments have increased the OS of patients with lung cancer and may control tumour-associated symptoms without adversely affecting the patients' overall quality of life (QoL) (4-6).

Prior to the development of epidermal growth factor receptor (EGFR)-tyrosine kinase inhibitor (TKI) therapy, chemotherapy with platinum-based doublets was administered to patients with stage IV non-small-cell lung cancer (NSCLC). Several meta-analyses have demonstrated the benefits of platinum-based doublet chemotherapy (7). The double-drug treatment strategy also results in improved OS rates compared with a single-drug chemotherapy regimen (8). Platinum-based doublets are usually combined with a third-generation cytotoxic drug, such as gemcitabine, vinorelbine or taxane; incorporation of pemetrexed and other drugs into individual treatment schedules may also be considered (9). Single- and double-drug chemotherapy has shown benefits in some elderly patients ( $>70$ years) (10). Compared with patients who received supportive care alone, patients treated with vinorelbine exhibited improved 1-year OS rates and a significantly improved QoL, with acceptable toxic effects (7). Furthermore, EGFR mutation (+) patients treated with EGFR-TKIs (such as, gefitinib, erlotinib or afatinib) exhibited a higher response rate, longer progression-free survival (PFS) and an improved QoL compared with patients treated with standard platinum-based doublet chemotherapy $(11,12)$.

The aim of lung cancer drug treatment is to control symptoms and improve the OS of patients. In Taiwan, platinum-based doublet chemotherapy and EGFR-TKIs are frequently used as a first-line treatment combined with third-generation cytotoxic combinations [Third generation Cytotoxic Combination (TCC), including paclitaxel, vinorelbine, gemcitabine and docetaxel], or as a monotherapy in select patients. A better suited drug treatment may be associated with improved outcomes. The aim of the present retrospective cohort study was to determine the real-world prescription rates of the various drugs used to treat patients with lung cancer in Taiwan and analyse the outcomes. 


\section{Materials and methods}

Data source and patient definition. The present study is a retrospective, population-based study using claims records from the entire National Health Insurance Database (NHID) of Taiwan between 2010 and 2015. NHID contains details of all the citizens in Taiwan (23,492,074 in 2015). NHID of Taiwan is a publicly available database through formal application and approved by the Health and Welfare Data Science Centre at The Ministry of Health and Welfare, Taiwan (https://dep.mohw.gov.tw/DOS/np-2500-113.html). The International Classification of Diseases, 9th Revision (ICD-9); Clinical Modification code (ICD-9 code 162) and catastrophic illness certificate (ICD-9 code 162) were used to select patients with lung cancer. This method of using a catastrophic illness certificate as the diagnostic criteria for lung cancer is strict and reliable. Patients who did not have a medical record of lung cancer with ambulatory (expenditures due to visits) and inpatient (expenditures due to admissions) care in the year 2010 were defined as lung cancer patients during 2011-2015 and were enrolled in this study as treatment-naive lung cancer patients. The TNM Staging of lung cancer and EGFR gene mutation status were identified according to the linked cancer registration file (13). Cancer registration file was founded in 1979. Hospitals with $>50$-bed capacity, which are able to provide care for outpatients and hospitalized patients with cancer were recruited and reported all newly diagnosed cases of malignant neoplasms to the registry. Staging and treatment details were required to report for specific cancers in 2002 and Site-specific factors (EGFR, Kras and other risk factors) were first included in 2012. Since the present study was between 2010 and 2015, information on EGFR mutation from certain patients may be missing.

Research ethics approval. The protocol used in the present study was approved by the Joint Institutional Review Board Taiwan R.O.C. (Protocol Number: 14-S-007). As this study was a retrospective database analysis study, it does not require informed consent according to local legislation issued by the Ministry of Health and Welfare in Taiwan (14).

Assessment. The primary objective of the present study was to determine the first-line drug treatment pattern in treatment-naive patients with lung cancer. The medications used for treating patients with lung cancer were in accordance with the Anatomical Therapeutic Chemical (ATC) classification (15). The use of antineoplastic and immunomodulating agents (ATC code, L) was monitored in these patients. The agents were categorized into five classes: Platinum-based compounds, third-generation cytotoxin combinations (paclitaxel, vinorelbine, gemcitabine and docetaxel; TCC), monotherapy (single third-generation cytotoxin; paclitaxel, vinorelbine, gemcitabine and docetaxel), EGFR-TKIs (afatinib, gefitinib and erlotinib) and others (other agents or combinations which were not included in the other groups). Following diagnosis of lung cancer, the first-line treatment was defined as the first anti-neoplastic agent prescription and its combination with other drugs within 90 days. Patients who underwent surgery were identified by their cancer registration file. The second-line treatment was identified as addition of one or more new anti-neoplastic agents following the first-line treatment. The code for neutropenia was ICD-9:288.0; for thrombocytopenia, ICD-9:287.4; Nausea and vomiting, ICD-9:787.0; neuropathy, ICD-9:357.3; rash, ICD-9:782.1; diarrhoea, ICD-9:787.9; nail disorders, ICD-9:703.8-9; and finger and toe disorders ICD-9:681.0-1.

Data analyses. SAS 9.1 (SAS Institute Inc.) was used for data analyses. The variable measures were identified based on the criteria described above. Frequencies/percentages were used to describe categorical variables. A log-rank test was used to compare the Kaplan-Meier curves from different treatment groups. OS for first- and second-line treatments were calculated from the first day of drug administration of first- and second-line treatments, respectively, until death. Cox regression analysis was used to identify the independent risk factors.

\section{Results}

Sample description. In 2015, the population of Taiwan was 23.4 million, of which 38,035 patients had lung cancer and there were 12,695 new cases of lung cancer in Taiwan. According to the National Health Insurance Database (NHID), lung cancer prevalence in Taiwan increased from 0.11 to $0.16 \%$ between 2010 and 2015, while the annual incidence density increased from 0.0524 to $0.0540 \%$ (Table I; Fig. 1).

First-line anti-neoplastic drug prescriptions in patients with lung cancer. Among the treatment-naive patients with lung cancer, 7,298-9,269 patients per year (60.42-71.99\%) were administered anti-neoplastic and immuno-modulating drugs (Fig. 2; Table SI). Platinum-based doublet chemotherapy was the most frequently prescribed first-line treatment $(47.75 \%$ in 2011 and $39.97 \%$ in 2015), followed by EGFR-TKI therapy (21.00\% in 2011 and $32.48 \%$ in 2015), other drugs ( 20\%), monotherapy (5.59\% in 2011 and $5.15 \%$ in 2015) and TCC therapy (0.71\% in 2011 and $0.37 \%$ in 2015) (Fig. 2; Table SI).

First-line clinical assessments. To determine the efficacy of different treatments and distinguish the patients' lung cancer stages, each patient's survival data was linked with their cancer registry file. Some of the cancer registration files lacked information, and as such, the final number of patients used for further staging and survival analysis was less than the total number of patients. Table II shows the patients' demographic data. A total of 38,100 patients underwent surgery or chemotherapy: Group $1(n=5,077)$, only surgery; group $2(n=7,392)$, surgery with chemotherapy; and group $3(n=25,631)$, only chemotherapy or EGFR-TKIs. In group 3, 10,588 patients underwent platinum-based doublet chemotherapy, 179 TCC therapy, 771 monotherapy, 8,008 EGFR-TKI therapy and 6,085 other treatments (Table II). Patients with stage I and II lung cancer in surgery and surgery plus chemotherapy showed significantly improved OS benefits compared with other treatment groups (Fig. 3A; $\mathrm{P}<0.001$ ). However, the surgery did not significantly improve OS in patients with stage IV lung cancer (Fig. 3C). EGFR-TKI therapy showed limited survival benefits for patients with stage I and II lung cancer, but compared to other treatments, OS was significantly increased with EGFR-TKI therapy in late-stage lung cancer (III and IV) (Fig. 3B and C). 


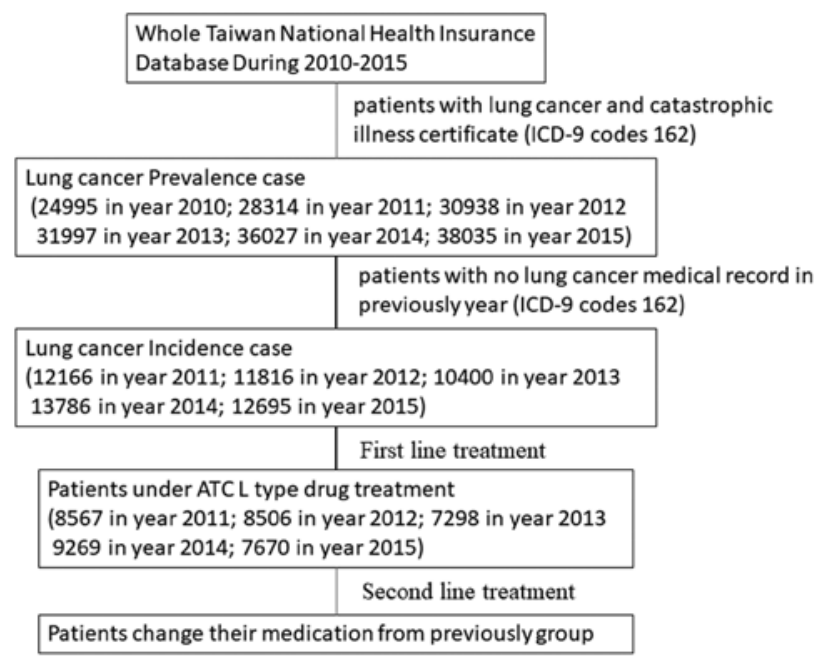

Figure 1. Selection and criteria of the cases used in the present study. ICD-9, International Classification of Diseases, 9th Revision; ATC, Alternative Therapeutic Chemical classification code; ATC-L, antineoplastic and immunomodulating agents.

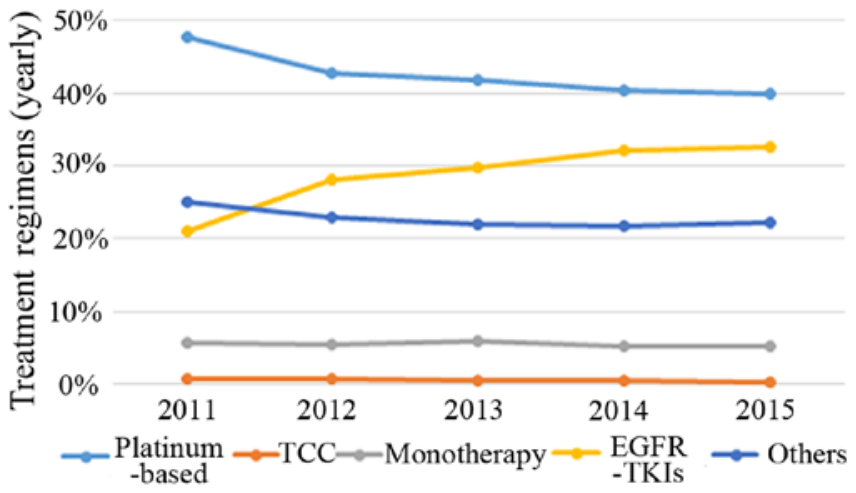

Figure 2. Percentage of patients treated with each of the various first-line treatments. The first line treatments were categorized into five groups: Platinum-based; TCC; monotherapy, EGFR-TKIs and others. EGFR-TKI, epidermal growth factor receptor-tyrosine kinase inhibitor. TCC, third-generation cytotoxin combinations (paclitaxel, vinorelbine, gemcitabine and docetaxel); monotherapy, single third-generation cytotoxin: Paclitaxel, vinorelbine, gemcitabine and docetaxel; EGFR-TKIs, afatinib, gefitinib and erlotinib; others, other agents or combination which did not fit into any of the categories.

The survival differences between the three EGFR-TKIs used (gefitinib, erlotinib and afatinib) were also determined. The OS rate for patients treated with gefitinib was significantly higher compared with erlotinib and platinum-based doublet chemotherapy (Fig. 3D). For afatinib, the sample size was too small to calculate the median survival rates. The detailed OS, median survival and hazard ratios are presented in Tables SII and SIII.

Results of the multivariate Cox hazards regression analysis for patients treated with different first-line treatments for lung cancer are shown in Table III. Overall, elderly patients with lung cancer (OR=1.01-1.03 compared with $<65)$ and those with late-stage lung cancer (OR=1.03-17.29 for stage IV compared with stage I; OR=0.65-4.16 for stage III compared with stage I; $\mathrm{OR}=0.79-2.42$ for stage II compared with stage I) had a poor OS, and women exhibited improved OS rates compared to men (OR=0.50-0.73 compared with men). 
Table II. Clinicopathological characteristics of patients treated with different first-line treatments for lung cancer. The EGFR expression status, lung cancer staging and grade were identified by linking with the cancer registration file.

\begin{tabular}{|c|c|c|c|c|c|c|c|}
\hline \multirow[b]{2}{*}{ Variable } & \multirow[b]{2}{*}{$\begin{array}{l}\text { Surgery } \\
\text { only (\%) }\end{array}$} & \multirow[b]{2}{*}{$\begin{array}{c}\text { Surgery plus } \\
\text { chemotherapy }(\%)\end{array}$} & \multicolumn{5}{|c|}{ Without surgery } \\
\hline & & & $\begin{array}{l}\text { Platinum-based } \\
\text { compounds }(\%)\end{array}$ & $\mathrm{TCC}(\%)$ & $\begin{array}{c}\text { Monotherapy } \\
(\%)\end{array}$ & $\begin{array}{c}\text { EGFR-TKIs } \\
(\%)\end{array}$ & Others $(\%)$ \\
\hline Patient number & 5,077 & 7,392 & 10,588 & 179 & 771 & 8,008 & 6,085 \\
\hline \multicolumn{8}{|l|}{ Sex } \\
\hline Male & $2,254(44.4)$ & $4,134(55.9)$ & $7,884(74.5)$ & $136(76.0)$ & $521(67.6)$ & $3,140(39.2)$ & $4,329(71.2)$ \\
\hline Female & $2,823(55.6)$ & $3,255(44.0)$ & $2,691(25.4)$ & $43(24.0)$ & $250(32,4)$ & $4,857(60.7)$ & $1,747(28.7)$ \\
\hline Unknown & & $3(0.1)$ & $13(0.1)$ & 0 & 0 & $11(0.1)$ & $9(0.1)$ \\
\hline \multicolumn{8}{|l|}{ EGFR mutation } \\
\hline Negative & $582(11.5)$ & $1,443(19.5)$ & $3,226(30.5)$ & $52(29.1)$ & $256(33.2)$ & $313(3.9)$ & $1,536(25.2)$ \\
\hline Positive & $742(14.6)$ & 2,213 (29.9) & 841 (7.9) & $4(2.2)$ & $62(8.0)$ & $6,801(84.9)$ & $349(5.8)$ \\
\hline Unknown & $3,753(73.9)$ & $3,736(50.6)$ & 6,521 (61.6) & $123(68.7)$ & $453(58.8)$ & 8,94 (11.2) & $4,200(69.0)$ \\
\hline \multicolumn{8}{|l|}{ TNM stage } \\
\hline 0 & $76(1.4)$ & $75(1.0)$ & $25(0.2)$ & 0 & $3(0.4)$ & $22(0.3)$ & $25(0.4)$ \\
\hline $\mathrm{I}$ & $3,827(75.4)$ & $2,812(38.0)$ & $298(2.8)$ & $7(3.9)$ & $64(8.3)$ & $184(2.3)$ & $344(5.7)$ \\
\hline II & 334 (6.7) & $1,070(14.5)$ & $321(3.0)$ & $12(6.7)$ & $35(4.5)$ & $85(1.1)$ & $318(5.2)$ \\
\hline III & $268(5.3)$ & $1,548(20.9)$ & 2,747 (25.9) & $42(23.5)$ & $141(18.3)$ & $612(7.6)$ & $1,195(19.6)$ \\
\hline IV & $210(4.1)$ & $1,707(23.1)$ & $6,989(66.0)$ & $118(65.9)$ & $504(65.4)$ & $6,972(87.1)$ & 3,965 (65.2) \\
\hline Unknown & $362(7.1)$ & $180(2.5)$ & $208(2.1)$ & & $24(3.1)$ & $133(1.7)$ & $238(3.9)$ \\
\hline \multicolumn{8}{|l|}{ Pathologic grade } \\
\hline 1 & $1,353(26.6)$ & $631(8.5)$ & $207(2.0)$ & $5(2.8)$ & $38(4.9)$ & $297(3.7)$ & $163(2.7)$ \\
\hline 2 & $2,392(47.1)$ & $3,034(41.0)$ & $1,508(14.2)$ & $23(12.8)$ & $170(22.0)$ & $1,544(19.3)$ & $919(15.1)$ \\
\hline 3 & $609(12.0)$ & $1,647(22.4)$ & $1,964(26.6)$ & $42(23.5)$ & $132(17.1)$ & $997(12.5)$ & $1,052(17.3)$ \\
\hline 4 & $60(1.2)$ & $104(1.4)$ & $71(0.7)$ & & $6(0.8)$ & $10(0.1)$ & $50(0.8)$ \\
\hline Other & $663(13.1)$ & $1,976(26.7)$ & $6,838(64.5)$ & $109(60.9)$ & $425(55.1)$ & $5,160(64.4)$ & $3,901(64.1)$ \\
\hline \multicolumn{8}{|l|}{ Area } \\
\hline North & $2,684(52.9)$ & $3,423(46.3)$ & $4,661(44.0)$ & $58(32.4)$ & $241(31.3)$ & $3,441(43.0)$ & $2,888(47.5)$ \\
\hline Central & $1,133(22.3)$ & $1,831(24.8)$ & $2,591(24.5)$ & $63(35.2)$ & $257(33.3)$ & $1,874(23.4)$ & $1,528(25.1)$ \\
\hline South & $1,154(22.7)$ & $1,941(26.3)$ & $2,990(28.2)$ & $53(29.6)$ & $256(33.2)$ & $2,453(30.6)$ & $1,450(23.8)$ \\
\hline East & $80(1.6)$ & $173(2.3)$ & $297(2.8)$ & $5(2.8)$ & $17(2.2)$ & $200(2.5)$ & $163(2.7)$ \\
\hline $\begin{array}{l}\text { Outer Islands } \\
\text { or unknown }\end{array}$ & $26(0.5)$ & $24(0.3)$ & $36(0.3)$ & & 0 & $40(0.5)$ & $56(0.9)$ \\
\hline \multicolumn{8}{|l|}{ Age } \\
\hline$<40$ & $133(2.6)$ & $152(2.1)$ & $243(2.3)$ & 0 & $14(1.8)$ & $122(1.5)$ & $42(0.7)$ \\
\hline $40-49$ & $513(10.1)$ & $703(9.5)$ & $1,016(9.6)$ & $7(3.9)$ & $36(4.7)$ & $584(7.3)$ & $122(2.0)$ \\
\hline $50-59$ & $1,310(25.8)$ & $1,842(24.9)$ & $2,535(23.9)$ & $17(9.5)$ & $101(13.1)$ & $1,586(19.8)$ & $402(6.6)$ \\
\hline $60-69$ & $1,565(30.8)$ & $2,295(31.0)$ & $3,311(31.3)$ & $41(22.9)$ & $137(17.8)$ & $1,920(24.0)$ & $831(13.7)$ \\
\hline $70-79$ & $1,174(23.1)$ & $1,883(25.5)$ & $2,754(26.0)$ & $78(43.6)$ & $295(38.3)$ & $2,251(31.9)$ & $2,088(34.3)$ \\
\hline$>80$ & $382(7.6)$ & $517(7.0)$ & $729(6.9)$ & $36(20.1)$ & $188(24.4)$ & $1,545(19.3)$ & $2,600(42.7)$ \\
\hline $\begin{array}{l}\text { Mean } \pm \text { standard } \\
\text { deviation }\end{array}$ & $62.9 \pm 11.6$ & $66.3 \pm 11.4$ & $63.4 \pm 11.3$ & $71.1 \pm 9.8$ & $70.3 \pm 12.2$ & $67.4 \pm 12.6$ & $75.7 \pm 10.8$ \\
\hline \multicolumn{8}{|l|}{ Side effects } \\
\hline Neutropenia & $21(0.4)$ & $510(6.9)$ & $1,505(14.2)$ & $11(6.1)$ & $30(3.9)$ & $303(3.8)$ & $247(4.1)$ \\
\hline Thrombocytopenia & $3(<0.1)$ & $24(0.3)$ & $63(0.6)$ & 0 & & $16(0.2)$ & $7(0.1)$ \\
\hline Nausea/vomiting & $67(1.3)$ & $589(8.0)$ & $1,058(10.0)$ & $4(2.2)$ & $32(4.2)$ & $627(7.8)$ & $192(3.2)$ \\
\hline Neuropathy & & $26(0.4)$ & $32(0.3)$ & 0 & 0 & $16(0.2)$ & $8(0.1)$ \\
\hline Rash & $6(0.1)$ & $50(0.7)$ & $74(0.7)$ & $8(4.5)$ & $3(0.4)$ & $92(1.1)$ & $13(0.2)$ \\
\hline Diarrhea & $29(0.6)$ & $234(3.2)$ & 303 (2.9) & & $20(2.6)$ & 367 (4.6) & 98 (1.6) \\
\hline Nail disorders & $3(<0.1)$ & $11(0.1)$ & $7(<0.1)$ & & 15 (1.9) & $22(0.3)$ & $3(<0.1)$ \\
\hline $\begin{array}{l}\text { Finger and toe } \\
\text { disorders }\end{array}$ & $14(0.3)$ & $183(2.5)$ & $117(1.1)$ & 0 & & $450(5.6)$ & $28(0.5)$ \\
\hline
\end{tabular}

EGFR, epidermal growth factor receptor; TKI, tyrosine kinase inhibitors; TCC, third-generation cytotoxin combinations (paclitaxel, vinorelbine, gemcitabine and docetaxel); TNM, Tumor-Node-Metastasis. 
A

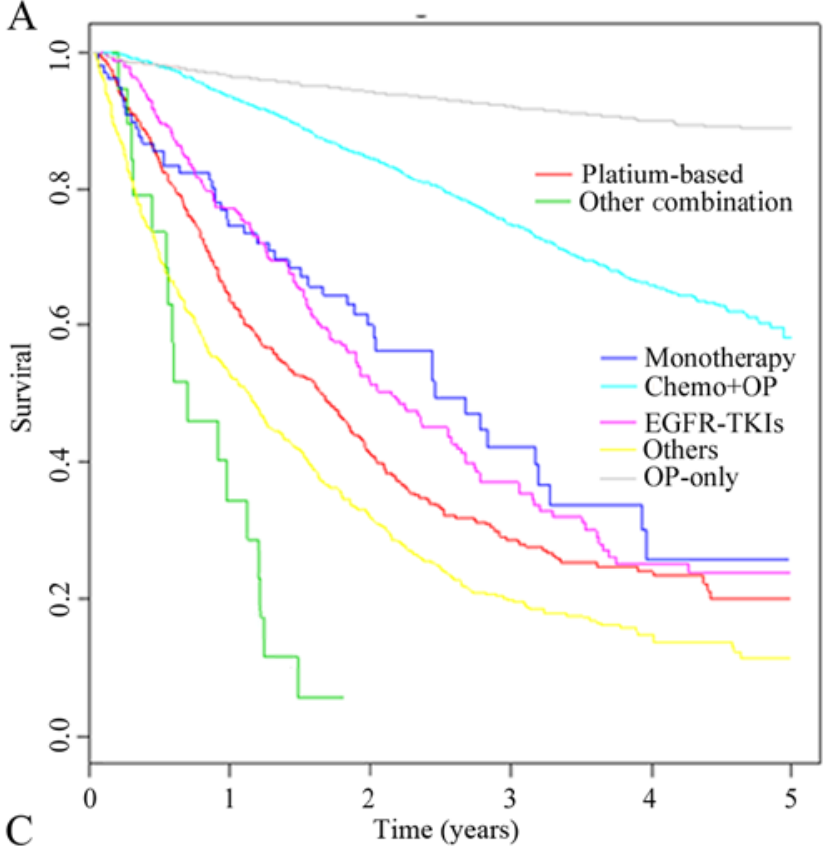

C

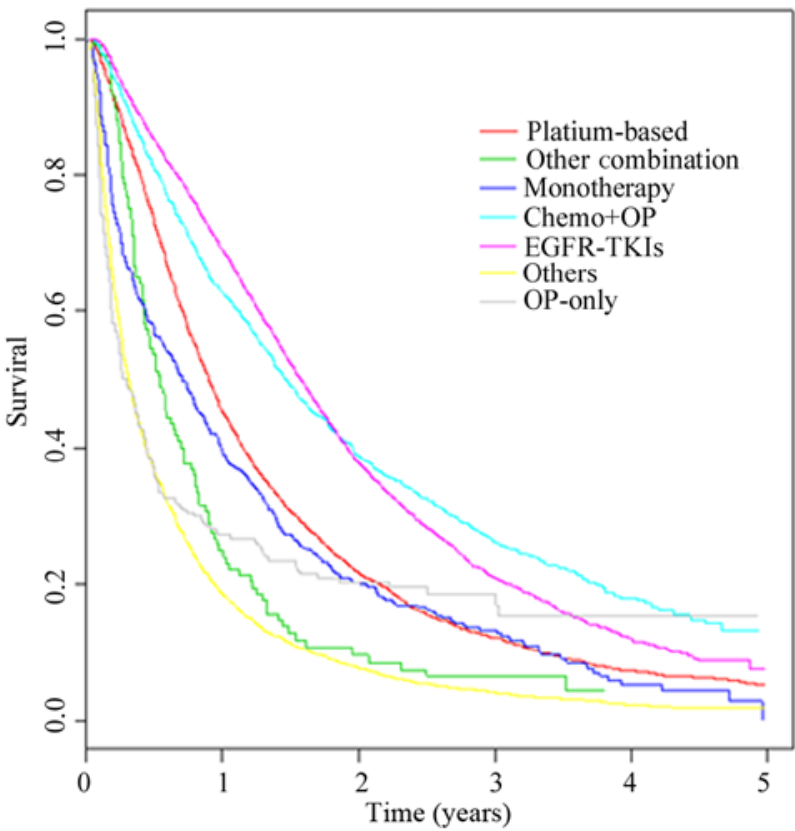

B

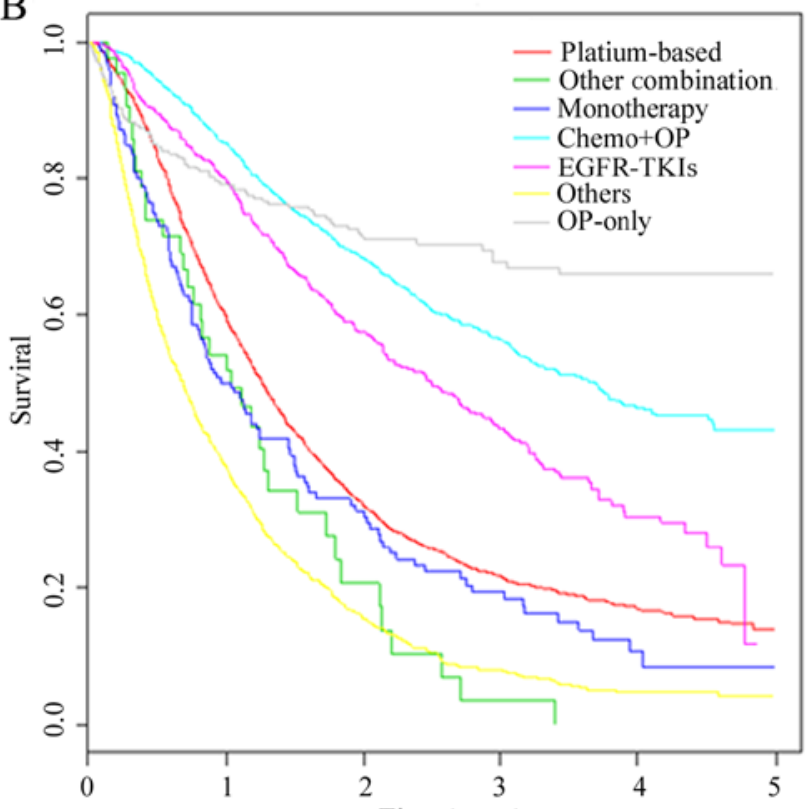

D

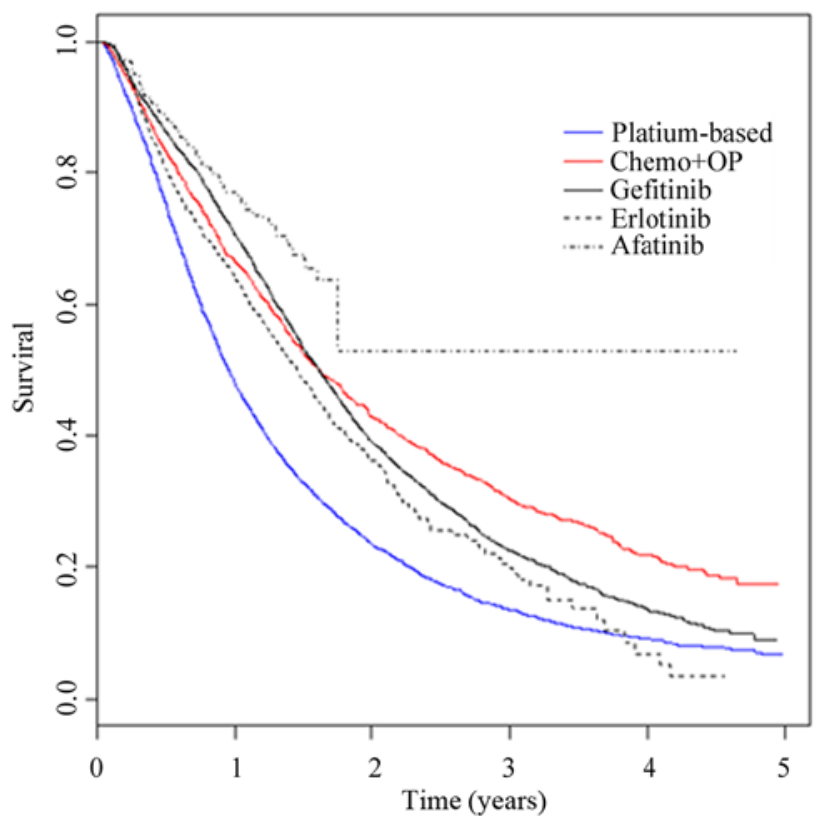

Figure 3. Kaplan-Meier survival time curves for patients with different stages of lung cancer and treated with various treatments. Overall survival of patients with (A) stage I and II, (B) stage III and (C) stage IV lung cancer treated with various first-line drug treatments. (D) Overall survival of patients with stage IIIB and IV lung cancer treated with various first-line drug treatments. Survival statistics are presented in Tables SII and SIII. EGFR-TKIs, afatinib, gefitinib and erlotinib; others, other agents which did not fit into any of the categories; Chemo, chemotherapy; OP, operation (surgery).

Efficacy of first-line treatment for patients with EGFR mutations. The EGFR mutation status was used to select patients with lung cancer who may exhibit an improved response to EGFR-TKI therapy. The activity of EGFR-TKIs in EGFR wild-type or unknown lung cancer patients still requires investigation. Patients with stage IIIB and IV lung cancer who underwent surgery with chemotherapy, platinum-based doublet chemotherapy or EGFR-TKI therapy were selected and their OS analysed. In patients with EGFR mutation (+), the OS was improved compared with patients with EGFR mutation (-) and EGFR mutation (unknown) in all the treatment groups (Fig. 4; Tables SIV and SV). The efficacies of platinum-based doublet chemotherapy and EGFR-TKI therapy were similar in EGFR mutation (+) patients (20.7 vs. 20.3 months; $\mathrm{P}=0.82$ ), whereas patients with EGFR mutation (-) who underwent platinum-based doublet chemotherapy had significantly improved OS compared with patients who underwent EGFR-TKI therapy (12.53 vs. 10.47 months; $\mathrm{P}=0.008$ ). In addition, EGFR mutation (unknown) patients who underwent EGFR-TKI therapy exhibited improved OS compared with patients who underwent platinum-based doublet chemotherapy (15.83 vs. 10.4 months; $\mathrm{P}<0.0001)$. These results suggest that EGFR-TKIs should not be used in patients with EGFR mutation (-).

Second-line clinical assessment. The benefits of different second-line treatments were assessed. Current guidelines 
recommend both chemotherapy and EGFR-TKIs, and the Taiwan National Health Insurance (NHI) system allows the use of both. Table IV shows the patients' demographic data. A total of 24,248 patients with lung cancer underwent second-line treatment. Pemetrexed $(n=4,962)$ for chemotherapy and erlotinib $(n=3,901)$ for targeted EGFR-TKI therapy were the most frequently prescribed. The median survival time for patients treated with gefitinib was 16.23 months, for pemetrexed it was 11.73 months and for the remainder of the drugs it was $\sim 7$ months (ranging between 6.73 and 8.2 months) (Fig. 5 and Tables SVI and SVII). These results suggest that as a second-line treatment, gefitinib resulted in improved OS compared with other chemotherapeutic drugs. Results of the multivariate Cox hazards regression analysis for different second-line treatments for lung cancer are shown in Table V. The efficacy of gefitinib was superior to other medication as second-line treatment for lung cancer. This result was similar for the first-line treatments.

\section{Discussion}

The present study is one of few retrospective cohort studies examining first-line treatment for treatment-naive lung cancer patients using a national sample (16). Using data from the NHID, it was demonstrated that first-line treatments used in Taiwan are similar to that recommended by the American Society of Clinical Oncology (ASCO) guidelines for patients with stage IV NSCLC (17). The majority of new cases of lung cancer are treated with platinum-based doublet chemotherapy or EGFR-TKI therapy as initial treatment (39.91 and 32.48\%, respectively, in 2015). Based on the data from NHID, the use of EGFR-TKIs for lung cancer treatment has increased over time, while that of other therapies has decreased. The results of the present study suggest that EGFR-TKIs as a first-line treatment is less efficacious compared with chemotherapy when used to treat patients with stage III and IV treatment-naive lung cancer. There is little benefit of treating patients with stage I and II lung cancer and no benefit for treating patients with stage III and IV with EGFR mutation (-) with EGFR-TKIs. In addition, direct comparison of EGFR-TKIs and other chemotherapy drugs used as second-line treatment showed that gefitinib was superior to the other drugs.

ASCO guidelines recommend platinum-based doublet chemotherapy for patients with NSCLC without EGFR or ALK mutations and with improved ability to take care of themself (17); however, a meta-analysis showed that 1-year survival was not significantly increased in patients treated with platinum-based doublet chemotherapy compared with patients treated with TCC therapy (18). Based on the data from NHID, median survival in patients with stage IV lung cancer who underwent platinum-based doublet chemotherapy was 11 months, which was higher compared with patients who underwent other types of chemotherapy (6.6 months with TCC therapy, 8.67 months with monotherapy and 3.77 months with others). This inconsistency in our results and previously published data may be due to different stage responses to various therapies, as the median survival in patients with stage III lung cancer was not significantly different between platinum-based doublet chemotherapy and TCC therapy [15.47 months (14.77-16.03) vs. 12.6 months (8.80-15.97)]. 

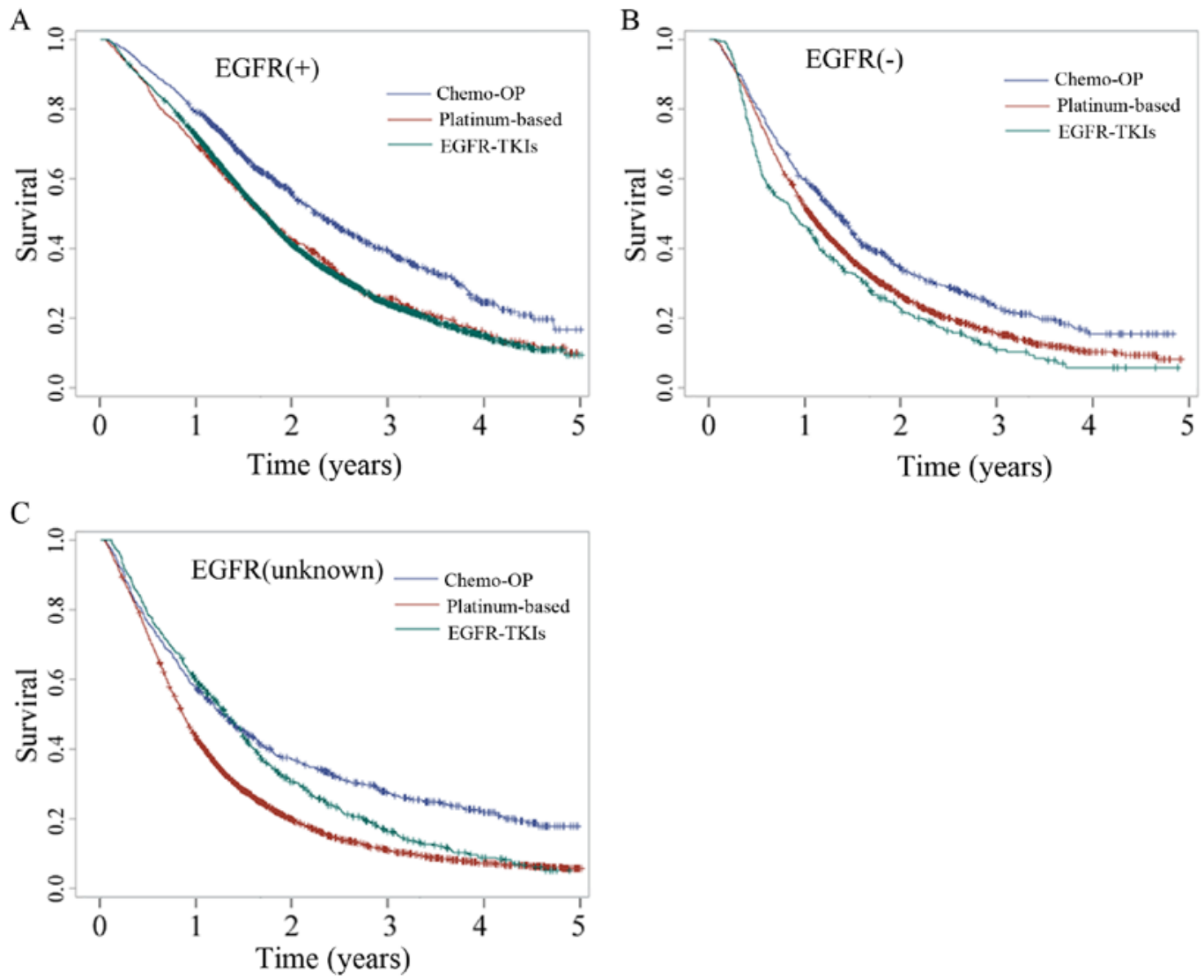

Figure 4. Kaplan-Meier survival curves of patients according to the EGFR Mutation status and treatment. Survival curves of patients with (A) EGFR Mutation (+), (B) EGFR Mutation (-) and (C) EGFR Mutation (unknown). Survival statistics are presented in Tables SIV and SV. EGFR-TKIs, afatinib, gefitinib and erlotinib; Chemo, chemotherapy; OP, operation (surgery).

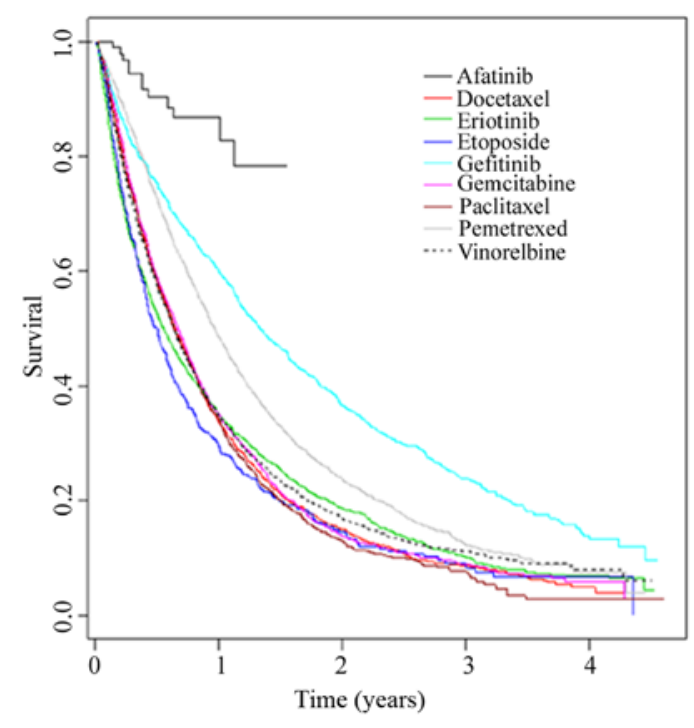

Figure 5. Kaplan-Meier survival curves of patients treated with various second-line anti-neoplastics. Three epidermal growth factor receptor-tyrosine kinase inhibitor agents, afatinib, gefitinib and erlotinib, and six chemotherapy agents, docetaxel, etoposide, gemcitabine, paclitaxel, pemetrexed, and vinorelbine were assessed. Survival statistics are presented in Tables SVI and SVII.

According to the Taiwan NHI system, patients with locally advanced NSCLC who fail to respond to chemotherapy as a first-line treatment (with or without EGFR mutation) are prescribed EGFR-TKIs. A few randomised studies comparing gefitinib with erlotinib prescription, showed that the median PFS in the gefitinib group was higher compared with the erlotinib group (4.9 months vs. 3.1 months; $95 \% \mathrm{CI}=1.3-8.5$ vs. 0.0-6.4) (19). In another cohort study in Taiwan, previously treated EGFR-TKIs naïve NSCLC patients administered with gefitinib had longer PFS and OS times compared with patients administered erlotinib (20). In the present study, OS for 9 commonly used second-line treatments for patients with lung cancer, three EGFR-TKIs (afatinib, gefitinib and erlotinib) and six chemotherapy drugs (docetaxel, etoposide, gemcitabine, paclitaxel, pemetrexed and vinorelbine) were assessed. Among the 24,292 lung cancer patients who received second-line treatment, 5,237 (21.56\%) were administered EGFR-TKIs. The results of the present study demonstrated that patients whom had previously been administered gefitinib had improved OS time. There are several possible explanations for the superior therapeutic effects of gefitinib compared with erlotinib. Erlotinib is more likely to be prescribed to patients with lung cancer with a higher severity of disease, such as those with cachexia and increased intracranial pressure (20). EGFR mutation status may also serve a crucial role in patients with lung cancer. Clinical evidence involving a comparison between chemotherapy and EGFR-TKIs as second-line treatment has yielded contrasting results which may be due to inconsistent inclusion criteria with, without, or with mixed EGFR mutation status (21-23). 


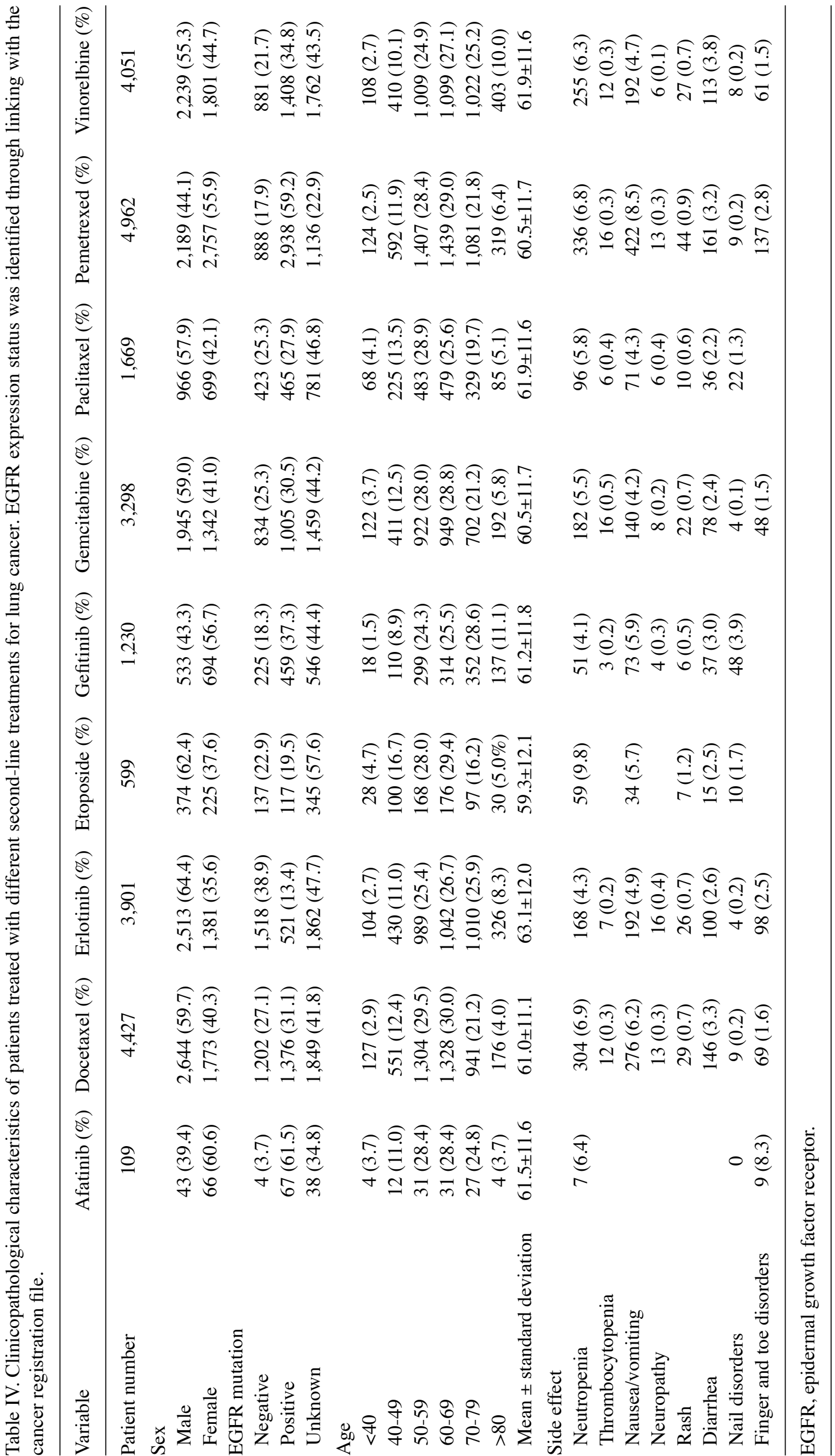




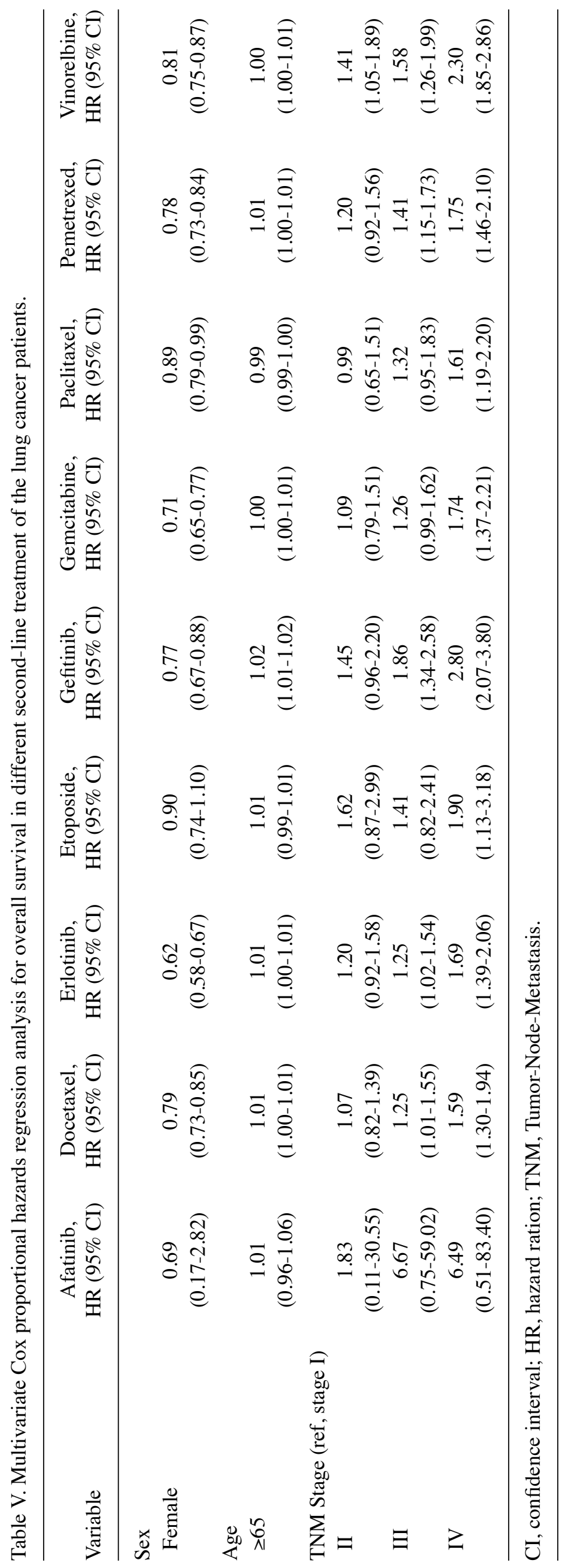


Claims-based analyses have several limitations, such as incomplete data and the possibility of coding errors or omissions. To better understand the information on lung cancer, data on lung cancer patients with catastrophic illness certificates (ICD-9 code 162) was linked with the cancer registry file (ICD-O-3, C33-C34, lung, bronchus and trachea). However, not all the relevant information was available on the initial staging of lung cancer. A high percentage $(>90 \%)$ of patients with stage IV lung cancer and treated with EGFR-TKI therapy as a first line treatment underwent molecular testing for EGFR mutation, and the majority of these tested positive. However, $<50 \%$ of patients with lung cancer undergoing platinum-based doublet chemotherapy underwent molecular testing to determine EGFR mutation status. Therefore, the results of the present study may not be generalizable, as lung cancer staging and EGFR gene mutation status may differ across countries. Performance status (PS) and smoking history may have an impact on outcomes and therapeutic strategies. There was no information on PS and smoking history listed in the cancer registry files. Drug-induced lung injuries was an adverse event during lung cancer treatment. There is no specific code for drug-induced lung injuries, including interstitial lung disease in ICD-9. The most similar coding was 'respiratory conditions due to other specified external drugs (508.0)'. To protect personal privacy, the Health and Welfare Data Science Centre only allows exporting results with more than two cases in each event. As there were results for patients with respiratory conditions with no more than two cases in each group, it was not possible to obtain and analyse this data. In future studies, data from medical charts may be used to examine the factors which may affect outcomes and therapeutic strategies.

The results of the present study suggested that patients with stage III or IV lung cancer undergoing first-line EGFR-TKI therapy may show improved OS; however, patients with stage I and II lung cancer may only exhibit smaller benefits and patients with stage III and IV EGFR mutation (-) patients may not benefit at all. The efficacy of two first-generation EFGR-TKIs may not be the same. Gefitinib may be more effective than erlotinib in treatment-naive and previously treated patients with lung cancer. Gefitinib also improved survival compared with other frequently used chemotherapy drugs. Additional randomised control trials are required to confirm this finding.

\section{Acknowledgements}

Not applicable.

\section{Funding}

The present study was supported by Mackay Medical College (Sanzhi, Taiwan; grant nos.MMC-1071E03 and MMC-1081B27).

\section{Availability of data and materials}

The present study is based on data from the Centre for Health and Welfare Data Science Centre in Ministry of Health and Welfare (H103065). The ownership of the data used in the present study belong to the National Health Insurance
Research Database (NHIRD) of Taiwan and cannot be made publicly available due to legal restrictions. However, the data are available through formal application to the Health and Welfare Data Science Centre at Ministry of Health and Welfare, Taiwan (https://dep.mohw.gov.tw/DOS/np-2500-113. html) and require a signed affirmation regarding data confidentiality. The authors have no special privilege of access to the database.

\section{Authors' contributions}

CC collected and analysed the data, and wrote the manuscript.

\section{Ethics approval and consent to participate}

The protocol used in the present study was approved by the Joint Institutional Review Board Taiwan R.O.C. (Protocol Number: 14-S-007).

\section{Patient consent for publication}

Not applicable.

\section{Competing interests}

The author declares that they have no competing interests.

\section{References}

1. Ferlay J, Steliarova-Foucher E, Lortet-Tieulent J, Rosso S, Coebergh JW, Comber H, Forman D and Bray F: Cancer incidence and mortality patterns in Europe: Estimates for 40 countries in 2012. Eur J Cancer 49: 1374-1403, 2013.

2. Siegel RL, Miller KD and Jemal A: Cancer statistics, 2015. CA Cancer J Clin 65: 5-29, 2015.

3. Wang BY, Huang JY, Cheng CY, Lin $\mathrm{CH}$, Ko J and Liaw YP: Lung cancer and prognosis in Taiwan: A population-based cancer registry. J Thorac Oncol 8: 1128-1135, 2013.

4. Douillard JY, Rosell R, De Lena M, Carpagnano F, Ramlau R, Gonzáles-Larriba JL, Grodzki T, Pereira JR, Le Groumellec A, Lorusso V, et al: Adjuvant vinorelbine plus cisplatin versus observation in patients with completely resected stage IB-IIIA non-small-cell lung cancer (Adjuvant Navelbine International Trialist Association [ANITA]): A randomised controlled trial. Lancet Oncol 7: 719-727, 2006.

5. Gilligan D, Nicolson M, Smith I, Groen H, Dalesio O, Goldstraw $P$, Hatton M, Hopwood P, Manegold C, Schramel F, et al: Preoperative chemotherapy in patients with resectable non-small cell lung cancer: Results of the MRC LU22/NVALT 2/EORTC 08012 multicentre randomised trial and update of systematic review. Lancet 369: 1929-1937, 2007.

6. Lim E, Harris G, Patel A, Adachi I, Edmonds L and Song F: Preoperative versus postoperative chemotherapy in patients with resectable non-small cell lung cancer: Systematic review and indirect comparison meta-analysis of randomized trials. J Thorac Oncol 4: 1380-1388, 2009.

7. NSCLC Meta-Analyses Collaborative Group: Chemotherapy in addition to supportive care improves survival in advanced non-small-cell lung cancer: A systematic review and meta-analysis of individual patient data from 16 randomized controlled trials. J Clin Oncol 26: 4617-4625, 2008.

8. Delbaldo C, Michiels S, Syz N, Soria JC, Le Chevalier T and Pignon JP: Benefits of adding a drug to a single-agent or a 2-agent chemotherapy regimen in advanced non-small-cell lung cancer: A meta-analysis. JAMA 292: 470-484, 2004.

9. Li M, Zhang Q, Fu P, Li P, Peng A, Zhang G, Song X, Tan M, Li X, Liu Y, et al: Pemetrexed plus platinum as the first-line treatment option for advanced non-small cell lung cancer: A meta-analysis of randomized controlled trials. PLoS One 7: e37229, 2012. 
10. Effects of vinorelbine on quality of life and survival of elderly patients with advanced non-small-cell lung cancer. The Elderly Lung Cancer Vinorelbine Italian Study Group. J Natl Cancer Inst 91: 66-72, 1999.

11. Maemondo M, Inoue A, Kobayashi K, Sugawara S, Oizumi S, Isobe H, Gemma A, Harada M, Yoshizawa H, Kinoshita I, et al: Gefitinib or chemotherapy for non-small-cell lung cancer with mutated EGFR. N Engl J Med 362: 2380-2388, 2010.

12. Han JY, Park K, Kim SW, Lee DH, Kim HY, Kim HT, Ahn MJ, Yun T, Ahn JS, Suh C, et al: First-SIGNAL: First-line single-agent iressa versus gemcitabine and cisplatin trial in never-smokers with adenocarcinoma of the lung. J Clin Oncol 30: 1122-1128, 2012.

13. Carter BW, Lichtenberger JP III, Benveniste MK, de Groot PM $\mathrm{Wu}$ CC, Erasmus JJ and Truong MT: Revisions to the TNM staging of lung cancer: Rationale, significance, and clinical application. Radiographics 38: 374-391, 2018.

14. http://regulation.cde.org.tw/data/downloadfile.php?sid=910

15. Skrbo A, Begovic B and Skrbo S: Classification of drugs using the ATC system (Anatomic, Therapeutic, Chemical Classification) and the latest changes. Med Arh 58 (1 Suppl 2): S138-S141, 2004 (In Bosnian).

16. Liang YH, Shao YY, Liao BC, Lee HS, Yang JC, Chen HM, Chiang CJ, Cheng AL and Lai MS: Cytotoxic chemotherapy as first-line therapy for advanced non-small-cell lung cancer in Taiwan: Daily practice. J Cancer 7: 1515-1523, 2016.

17. Masters GA, Temin S, Azzoli CG, et al: Systemic therapy for stage IV non-small-cell lung cancer: American Society of Clinical Oncology Clinical Practice Guideline Update. J Clin Oncol 33: 3488-3515, 2015.

18. D'Addario G, Pintilie M, Leighl NB, Feld R, Cerny T and Shepherd FA: Platinum-based versus non-platinum-based chemotherapy in advanced non-small-cell lung cancer: A meta-analysis of the published literature. J Clin Oncol 23: 2926-2936, 2005.
19. Kim ST, Uhm JE, Lee J, Sun JM, Sohn I, Kim SW, Jung SH, Park YH, Ahn JS, Park K and Ahn MJ: Randomized phase II study of gefitinib versus erlotinib in patients with advanced non-small cell lung cancer who failed previous chemotherapy. Lung Cancer 75: 82-88, 2012.

20. Chang CH, Lee CH, Ko JC, Chang LY, Lee MC, Wang JY and Yu CJ: Gefitinib or erlotinib in previously treated non-small-cell lung cancer patients: A cohort study in Taiwan. Cancer Med 6: $1563-1572,2017$.

21. Kim ES, Hirsh V, Mok T, Socinski MA, Gervais R, Wu YL, Li LY, Watkins CL, Sellers MV, Lowe ES, et al: Gefitinib versus docetaxel in previously treated non-small-cell lung cancer (INTEREST): A randomised phase III trial. Lancet 372: 1809-1818, 2008.

22. Ciuleanu T, Stelmakh L, Cicenas S, Miliauskas S, Grigorescu AC, Hillenbach C, Johannsdottir HK, Klughammer B and Gonzalez EE: Efficacy and safety of erlotinib versus chemotherapy in second-line treatment of patients with advanced, non-small-cell lung cancer with poor prognosis (TITAN): A randomised multicentre, open-label, phase 3 study. Lancet Oncol 13: 300-308, 2012.

23. Garassino MC, Martelli O, Broggini M, Farina G, Veronese S, Rulli E, Bianchi F, Bettini A, Longo F, Moscetti L, et al: Erlotinib versus docetaxel as second-line treatment of patients with advanced non-small-cell lung cancer and wild-type EGFR tumours (TAILOR): A randomised controlled trial. Lancet Oncol 14: 981-988, 2013. International (CC BY-NC-ND 4.0) License. 